\title{
Functionalization of Au and Ag Nanoparticles with Novel Organodichalcogenide compounds
}

\author{
Leandro M. S. Takata (PG) ${ }^{\star}$, Augusto C. Gonçalves (PG), Pedro H. C. Camargo (PQ), \\ Alcindo A. Dos Santos (PQ)
}

Instituto de Química - Universidade de São Paulo. *takata@iq.usp.br

Keywords: Organodichalcogenides, Gold Nanoparticles, Silver Nanoparticles.

\section{INTRODUCTION}

Gold (Au) and silver (Ag) nanoparticles (NPs) functionalized with organochalcogenides of selenium (Se) and tellurium ( $\mathrm{Te}$ ) are very attractive towards the search of new diagnostic tools, biosensors and organocatalists. ${ }^{1}$ However, these systems are much less investigated compared to its sulfur analogues. ${ }^{2}$ In this study, we aim at the synthesis of a variety of dichalcogenides based on Se and Te containing amino acids groups followed by their utilization to prepare organoselenium and organotellurium functionalized Au and Ag NPs.

\section{RESULTS AND DISCUSSION}

The $\mathrm{Au}$ and $\mathrm{Ag}$ NPs were prepared by reduction of $\mathrm{HAuCl}_{4}$ or $\mathrm{AgNO}_{3}$ using sodium citrate solution at $100{ }^{\circ} \mathrm{C}$. The organochalcogenides were synthesized via the nucleophilic substitution of halides by dilithium dichalcogenolate, which was generated from elemental chalcogen reduction using lithium triethylborohydride, according scheme 1.

$$
\begin{aligned}
& \mathrm{Y}^{0} \stackrel{\mathrm{LiEt}_{3} \mathrm{BH}}{\underset{1 \text { eq. }}{\longrightarrow}}\left[\mathrm{Li}_{2} \mathrm{Y}_{2}\right] \stackrel{\mathrm{HO} \sim \mathrm{Br}_{\mathrm{Br}}}{\longrightarrow} \mathrm{HO}_{\mathrm{Y}}{ }^{-\mathrm{Y}} \sim_{\mathrm{OH}} \\
& \text { 1a: } Y=\operatorname{Se}(72 \%) \\
& \text { 1b: } Y=\mathrm{Te}(43 \%)
\end{aligned}
$$

Scheme 1. Preparation of organic dichalcogenides.

The hydroxyl group present at the both ends of the molecule enabled it to be coupled with different amino acids, leading to the formation of more

$$
\begin{aligned}
& \text { 2a: } Y=\operatorname{Se}(80 \%) \\
& \text { 2b: } Y=\operatorname{Te}(78 \%)
\end{aligned}
$$
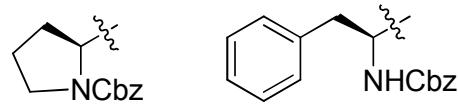

After their synthesis, we focused on the functionalization of $\mathrm{Au}$ and $\mathrm{Ag}$ NPs with the produced organodichalcogenides (2-4, a and b). In a typical protocol, 2-4 ( $\mathbf{a}$ and $\mathbf{b}$ ) were added into an ethanolic suspension containing the Au or Ag NPs. Next, this mixture was stirred at room temperature for $6 \mathrm{~h}$. Finally, the functionalized NPs were isolated by centrifugation and washed with ethanol.

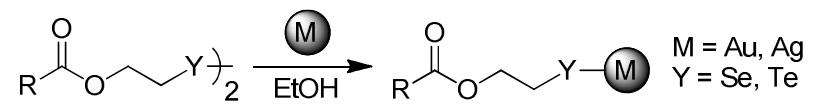

Scheme 3. Functionalization of $\mathrm{Ag}$ and $\mathrm{Au}$ NPs

The functionalized NPs ( $\mathbf{M}-\mathbf{X a}$ and $\mathbf{M}-\mathbf{X b}$, where $\mathbf{M}=$ $\mathrm{Ag}$ and $\mathrm{Au}$ and $\mathbf{X}=1,2,3$ and 4) were analyzed by surface-enhanced Raman spectroscopy. Our results showed that the functionalization was successfully achieved for the organodisselenides onto Ag NPs (Ag-2a, Ag-3a and Ag-4a) and organotellurides onto $\mathrm{Au}$ NPs (Au-2b, Au-3b and $\mathbf{A u - 4 b}$ ). In these cases, the recorded spectra suggest that the functionalization occurred with the cleavage of the chalcogen-chalcogen bond during the binding process. On the other hand, typical signals due to oxidation at the surface of the Ag NPs could be detected in the spectra when the $\mathbf{2} \mathbf{b}, \mathbf{3} \mathbf{b}$ and $\mathbf{4 b}$ were used for functionalization. Also, no signals could be detected after functionalization of $\mathrm{Au}$ NPs with compounds $\mathbf{2 a}, \mathbf{3} \mathbf{b}$ and $\mathbf{4 a}$ (orgadisselenides).

\section{CONCLUSION}

Our results show that the functionalization of $\mathrm{Au}$ and Ag NPs with the novel organodichalcogenides occurred successfully only for organodisselenides onto Ag NPs and organoditellurides onto Au NPs. A complete characterization of compounds $\mathbf{2 - 4}$, $\mathbf{a}$ and $\mathbf{b}$ as well as the products obtained by their functionalization on Au and Ag NPs are in progress in our laboratory.

elaborated structures as shown in Scheme 2.

Scheme 2. Preparation of dichalcogenides containing amino acid goups.

\section{ACKNOWLEDGEMENTS}

FAPESP, CAPES, CNPq, IQ-USP.

\section{REFERENCES}

${ }^{1}$ a) de la Llave, E,; Scherlis, D. A.; Langmuir 2010, 26, 173. b) Ulman, A. Chem. Rev., 1996, 96, 1553.

${ }^{2}$ Love, J. C.; Estroff, L. A.; Kriebel, J. K.; Nuzzo, R. G.; Whitesides, G. M. Chem. Rev. 2005, 105, 11. 